\title{
La persona del terapeuta: Validación española del Cuestionario de Evaluación del Estilo Personal del Terapeuta (EPT-C)
}

\author{
Javier Prado-Abril ${ }^{1}$, Javier Fernández-Álvarez ${ }^{2}$, Sergio Sánchez-Reales ${ }^{3,4}$, Soo Jeong Youn ${ }^{5}$ \\ Félix Inchausti ${ }^{6}$ y Guadalupe Molinari ${ }^{7}$ \\ ${ }^{1}$ Servicio Aragonés de Salud, IIS Aragón, Zaragoza, España \\ ${ }^{2}$ Università Cattolica del Sacro Cuore, Milán, Italia \\ ${ }^{3}$ Servicio Murciano de Salud, Murcia, España \\ ${ }^{4}$ Universidad Internacional de la Rioja, Logroño, España \\ ${ }^{5}$ Massachusetts General Hospital, Harvard Medical School, Boston, MA, United States \\ ${ }^{6}$ Servicio Riojano de Salud, Logroño, España \\ ${ }^{7}$ Universitat Jaume I, Castellón, España
}

\begin{abstract}
The person of the therapist: Spanish validation of the Personal Style of the Therapist Questionnaire (PST-Q). The Personal Style of the Therapist Questionnaire (PST-Q) is an instrument with more than 20 years of theoretical and empirical developments at the international scenario, especially in Latin America. The main goal of the study was to provide preliminary evidence on its psychometric properties and factorial structure in a sample of Spanish licensed clinical psychologists $(N=350)$. 7 confirmatory factor analysis were performed to found a 20-item and 5-factor solution that had the best fit indices and internal consistency values. The original abbreviated model of the PST-Q was partially confirmed, since item 28 had to be eliminated because it had a factor weight less than .30 . The alpha values were slightly lower than the original, but equivalent to those of the Portuguese validation. The implications of the findings for training and research in psychotherapy and clinical psychology are discussed.
\end{abstract}

Keywords: Personal style of the therapist; PST-Q; practice-oriented research; clinical psychology; psychotherapy.

Resumen: El Cuestionario de Evaluación del Estilo Personal del Terapeuta (EPT-C) es un instrumento con más de 20 años de desarrollos teóricos y empíricos a nivel internacional, especialmente en Latinoamérica. El objetivo del estudio consistió en proporcionar evidencia preliminar sobre sus propiedades psicométricas y estructura factorial en una muestra de psicólogos clínicos españoles debidamente acreditados $(N=350)$. Se realizaron 7 análisis factoriales confirmatorios hasta dar con una solución de 5 factores y 20 ítems que resultó ser la que presentó los mejores índices de ajuste y de consistencia interna. Se confirmó parcialmente el modelo abreviado original del EPT-C, ya que tuvo que eliminarse el ítem 28 por presentar una carga factorial inferior a .30. Los valores alfa fueron ligeramente inferiores al original, pero equivalentes a los de la validación portuguesa. Se discuten las implicaciones de los hallazgos para la formación e investigación en psicoterapia y psicología clínica.

Palabras clave: Estilo personal del terapeuta; EPT-C; investigación orientada por la práctica; psicología clínica; psicoterapia.

Recibido: 11 de mayo 2019; aceptado: 11 de diciembre 2019. Correspondencia: Javier Prado-Abril. Servicio Aragonés de Salud, IIS Aragón, Zaragoza, España. Correo-e: jpradoabril@gmail.com Agradecimientos: Este trabajo no hubiera sido posible sin la decisiva colaboración de la Asociación Nacional de Psicólogos Clínicos y Residentes (ANPIR), la Sociedad de Psicología Clínica Asturiana (SOPCA) y la Asociación Galega de Psicólogos Internos Residentes (AGAPIR). Estas sociedades garantizan que la muestra de este estudio reúne las características que habilitan para el ejercicio de la psicología clínica en España (Real Decreto 2490/1998; Ley 44/2003).

\section{Introducción}

Los resultados de los pacientes que acuden a psicoterapia se explican por la interrelación de un conjunto diverso de factores. La conocida clasificación de Lambert (2013) recoge la relevancia de los factores técnicos, los factores comunes, las expectativas y los aspectos extra-terapéuticos. Asimismo, el avance de las últimas décadas en investigación en psicoterapia cristaliza en cier- 
to acuerdo en torno a la efectividad general de la psicoterapia (American Psychological Association [APA], 2013), aunque el gran debate de la psicoterapia sigue vigente (Hofmann y Barlow, 2014; Laska, Gurman y Wampold, 2014; Wampold y Imel, 2015). No obstante, este consenso favorece el avance de la investigación de aquellas características de pacientes y terapeutas que contribuyen al resultado final de la psicoterapia (Barkham, Lutz, Lambert y Saxon, 2017; Bohart y Wade, 2013; Prado-Abril, Gimeno-Peón, Inchausti y Sánchez-Reales, 2019) y, en definitiva, a la investigación centrada en el proceso psicoterapéutico. Por ejemplo, en la revisión de Barkham et al. (2017) se señala que los efectos del terapeuta explican alrededor del $8 \%$ de la varianza de los resultados. En consecuencia, estos autores resaltan la importancia de incrementar los esfuerzos de la investigación centrada en variables que, como los efectos del terapeuta, contribuyen al cambio de los pacientes.

Una forma de acercarse al estudio de los efectos del terapeuta es tratar de averiguar cómo desarrollan su práctica los psicólogos clínicos: descubrir los actos y procederes habituales de los clínicos en sesión (Fernández-Álvarez, Castañeiras, García, Gómez y Fernández-Álvarez, 2017; Prado-Abril, Sánchez-Reales, y Inchausti, 2017). En este sentido, el estilo del terapeuta es un tópico con cierta tradición en el campo de la psicoterapia. Entre las aproximaciones clásicas más influyentes se encuentran la de Orlinsky, Grawe y Parks (1994) que lo definen como aquellos rasgos de la personalidad del terapeuta que se expresan en el proceso psicoterapéutico influyendo en la alianza terapéutica y en los resultados del tratamiento. Por su parte, Beutler et al. (2004) lo definen como un estado objetivo estable en el tiempo y a través de diferentes situaciones que modula los procedimientos que se implementan. Con estos antecedentes se ha desarrollado un constructo diferente, conocido como Estilo Personal del Terapeuta (EPT), que integra las nociones anteriores y cuenta con más de 20 años de desarrollos teóricos y empíricos desde su formulación inicial (Casari, Ison, y Gómez, 2018; Fernández-Álvarez, García, y Scherb, 1998). El EPT se define como el conjunto de características que cada terapeuta aplica en cada situación psicoterapéutica, constituyendo sus atributos básicos. Comprende aspectos singulares e idiosincrásicos y aquellas condiciones que empujan a los clínicos a comportarse de un modo particular en el transcurso de su desempeño profesional (Fernández-Álvarez, García, Lo Bianco, y Corbella, 2003; Fernández-Álvarez et al., 1998). Por lo tanto, aunque el EPT se relaciona con el modelo teórico de pertenencia y determina la selección de técnicas específicas a implementar en el curso de un tratamiento psicológico, también implica un sello personal (Fernández-Álvarez et al., 2017).

El constructo EPT se ha operacionalizado principalmente a través del Cuestionario de Evaluación del Estilo Personal del Terapeuta (EPT-C). Un auto-informe compuesto por 36 ítems, a completar mediante un sistema de respuesta likert de 7 puntos, cuya validación preliminar se realizó sobre una muestra de psicoterapeutas argentinos a través de dos estudios factoriales (Fernández-Álvarez, García, Castañeiras, y Rial, 2005; Fernández-Álvarez et al., 2003). Posteriormente, se desarrollaron una versión breve de 21 ítems (Castañeiras, Ledesma, García, y Fernández-Álvarez, 2008) y adaptaciones satisfactorias para población chilena, brasileña y portuguesa (Casari et al., 2018). La versión original organiza las puntuaciones en 5 dimensiones o funciones. La función Instruccional se relaciona con el establecimiento del encuadre psicoterapéutico y sus puntuaciones se ordenan de menor a mayor en un continuo que oscila entre la flexibilidad y la rigidez. La función Expresiva versa sobre el estilo de comunicación emocional del terapeuta incluyendo aspectos como priorizar el trabajo con las emociones o el uso de las auto-revelaciones como recurso psicoterapéutico. Puntuaciones bajas implican una presencia distante y formal, mientras que puntuaciones elevadas representan una mayor proximidad. La función Involucración describe el nivel de compromiso del terapeuta en su trabajo, evaluando aspectos como la capacidad para que los terapeutas desconecten de las circunstancias de sus pacientes entre sesiones. El continuo oscila entre baja y alta involucración. La función Atencional define el estilo o modo preferido de escuchar, explorar y obtener información clínica. Puntuaciones bajas se interpretan como atención abierta y puntuaciones elevadas como focalizada. Finalmente, la dimensión Operativa se relaciona con el modo de proceder e intervenir a nivel técnico e implica cuestiones como la planificación y el grado de estructuración del trabajo en sesión. El continuo, de menor a mayor puntuación, se mueve entre un estilo espontáneo y otro pautado y estructurado. Las últimas investigaciones con el EPT-C se han centrado en su validez de constructo y en dotarlo de validez convergente con un sistema de observación y codificación de la conducta verbal de los psicoterapeutas (Fernández-Álvarez et al., 2015a). Esto permite, mediante un criterio externo objetivo, verificar la precisión de las auto-descripciones de los clínicos que completan el cuestionario. Por ejemplo, en un estudio reciente, que analizó las grabaciones de 79 sesiones conducidas por 20 psicoterapeutas, se relacionaron las dimensiones atencional y operativa con diferentes perfiles de actuación verbal (Fernández-Álvarez et al., 2017). 
Diferentes trabajos empíricos han relacionado el constructo EPT con variables relevantes en el proceso psicoterapéutico (Casari et al., 2018). Entre las observaciones más destacadas se encuentran las relaciones que guarda el EPT con la experiencia (medida como años de ejercicio profesional) y con la orientación teórico-técnica (auto-informada por los psicoterapeutas) (p.ej., Castañeiras, García, Lo Bianco, y Fernández-Álvarez, 2006). Con los años de ejercicio se han vinculado, especialmente, las funciones atencional y operativa. Con independencia de la orientación teórico-técnica, una mayor experiencia se relaciona con una atención más abierta y menos focalizada, con un proceder más flexible y espontáneo, y con el uso de un menor número de preguntas y más dirigidas a la comprensión de la experiencia subjetiva de los pacientes (Castañeiras et al., 2006; Fernández-Álvarez et al., 2015a; 2017; Gómez et al., 2011). En suma, parece que los clínicos, al inicio de su práctica independiente, tienden a ser más directivos: muestran una atención más focalizada, son más propensos a adherirse a protocolos pautados de tratamiento y realizan mayor cantidad de preguntas y más centradas en hechos que en la naturaleza de la relación terapéutica y la experiencia subjetiva de los pacientes. Si bien los estudios no profundizan en la explicación de estas observaciones, podría hipotetizarse que la adherencia a protocolos de tratamiento reduce la incertidumbre y dota al novel de cierta seguridad (Prado-Abril, Sánchez-Reales, y García-Campayo, 2016). En cualquier caso, estos estudios sugieren, con las limitaciones propias de los estudios correlacionales, que el EPT se rige por la misma dialéctica entre estabilidad y cambio que otras dimensiones de la personalidad, convirtiéndolo en una variable interesante y susceptible de ser tomada en consideración dentro de la formación sanitaria especializada en Psicología Clínica (Prado-Abril et al., 2017; 2019).

En cuanto a las relaciones entre el EPT y el modelo teórico-técnico de pertenencia existen algunos estudios de sumo interés. En el estudio de Lee, Neimeyer y Rice (2013), sobre una muestra compuesta por 1151 psicoterapeutas, se encontró que la posición epistemológica (racionalista o constructivista) determinaba el EPT. En concreto, los psicoterapeutas racionalistas u objetivistas mostraron una menor involucración, mayor atención focalizada y una orientación operativa más pautada. Por su parte, se ha encontrado que los terapeutas psicoanalistas tendrían menores puntuaciones en las funciones atencional, operativa, expresiva y de involucración (Casari, Albanesi y Maristany, 2013; Castañeiras et al., 2008). Lo cual es coherente con algunos modelos psicoanalíticos tradicionales que enfatizan la prescripción de dejar la atención flotante al inicio de la sesión y guiar mínima- mente el análisis del paciente. Tampoco sorprenden los resultados de Vides-Porras, Grazioso y García de la Cadena (2012) que sugieren un estilo más directivo por parte de terapeutas cognitivo-conductuales en comparación con terapeutas integradores. Finalmente, recientemente, Quiñones, Ugarte, Ceric, García y Santibañez (2019) encuentran también diferencias entre clínicos que se adhieren a una orientación cognitiva post-racionalista respecto a otros de orientación sistémica. En resumen, estos estudios explicitan que a través del EPT de los clínicos se pueden rastrear sus coordenadas teóricas de referencia. No obstante, estos estudios no indagan si es el estilo personal lo que determina la elección formativa en una escuela psicoterapéutica $o$, por el contrario, es la propia formación la que modula el estilo personal. Aunque probablemente se trate de una interrelación de factores que activan diversos procesos sucesivos que se desarrollan en la práctica clínica con pacientes. Es decir, ciertas características personales de los clínicos los inclinan hacia cierta dirección de intereses y, a su vez, estos intereses actualizan o refuerzan ciertas características estilísticas en el desarrollo del ejercicio de la psicoterapia. Como se verá en el apartado de la descripción de la muestra (Tabla 1), que la integración sea la norma entre los clínicos españoles no debería resultar llamativo. En nuestro contexto, el ejercicio de la Psicología Clínica exige dominar multiplicidad de ámbitos de actuación, donde las características de los pacientes implican la totalidad del espectro de gravedad representando una situación que, no es aventurado señalar, empuja a nuestros clínicos a ser flexibles, prácticos y creativos. Los modelos de escuela tienen sus virtudes, pero en ocasiones su generalización a cualquier circunstancia clínica no se incluye entre ellas (Norcross y Goldfried, 2005).

Recientemente, en nuestro entorno y en el contexto de la formación sanitaria especializada vía PIR (Psicólogo Interno Residente), se está trabajando en modelos de entrenamiento y supervisión en psicoterapia para residentes (Prado-Abril et al., 2017; 2019). En este ámbito, el EPT-C se revela como un instrumento de evaluación que puede ser de mucha utilidad en la personalización de la formación y supervisión de los futuros psicólogos clínicos. Del mismo modo que se recomienda monitorizar sistemáticamente la evolución de nuestros pacientes para ajustar la intervención a las necesidades del caso (Gimeno-Peón, Barrio-Nespereira y Prado-Abril, 2018), parece lógico hacerlo también con la progresión como psicoterapeutas de los residentes bajo supervisión. Por otro lado, aunque el EPT-C es un instrumento psicométricamente consistente, existen motivos que justifican la revisión de sus propiedades psicométricas y su estructura factorial en una muestra de psicólogos clínicos espa- 
ñoles. En primer lugar, las diferencias culturales que pueden existir entre España y Argentina; y, en segundo lugar, la idiosincrasia de los psicólogos clínicos españoles que representan una población muy particular que comparte un estándar de calidad definido por un órgano ministerial que los regula y certifica como tales (Real Decreto 2490/1998; Ley 44/2003). Por ende, el objetivo de este trabajo fue determinar las propiedades psicométricas del EPT-C en una muestra de 350 psicólogos clíni$\cos$, con la intención de que pueda convertirse en un recurso de calidad para la formación y la investigación en nuestro singular contexto profesional y cultural.

\section{Método}

\section{Participantes}

La muestra estuvo constituida por 350 psicólogos clínicos españoles, 271 mujeres $(77.4 \%)$ y 79 hombres (22.6\%), con edades comprendidas entre los 24 y los 68 años $(M=36.67$ y $D T=7.67)$ y una experiencia media de 9.85 años $(D T=8.05)$. El $21.4 \%$ se encontraban realizando la residencia PIR y el $78.6 \%$ eran especialistas. Respecto a la orientación teórica el $67.1 \%$ se definieron como integradores, un $17.7 \%$ como cognitivo-conductuales, un $9.4 \%$ como sistémicos, un $4.6 \%$ como psicodinámicos y un $1.2 \%$ como humanista-existenciales (Tabla 1).

Tabla 1. Estadísticos descriptivos de la muestra

\begin{tabular}{lc}
\hline & $n=350$ \\
\hline Mujeres (\%) & $271(77.4)$ \\
Edad, $M(D T)$ & $36.67(7.67)$ \\
Experiencia clínica, $M(D T)$ & $9.85(8.05)$ \\
Residentes (\%) & $75(21.4)$ \\
Especialistas (\%) & $275(78.6)$ \\
Orientación teórica (\%) & \\
$\quad$ Integración & $235(67.1)$ \\
$\quad$ Cognitivo-Conductual & $62(17.7)$ \\
$\quad$ Sistémica & $33(9.4)$ \\
Psicodinámica & $16(4.6)$ \\
$\quad$ Humanista-Existencial & $4(1.2)$ \\
\hline
\end{tabular}

Notas: La experiencia clínica se mide como años de ejercicio e incluye los años como residente. La integración incluye tanto la integración teórica como el eclecticismo técnico. La orientación cognitivo-conductual incluye los modelos de primera, segunda y tercera generación.

\section{Instrumentos}

Cuestionario de Evaluación del Estilo Personal del Terapeuta (EPT-C; Fernández-Álvarez et al., 2003). Auto-informe de 36 ítems que se responde con una escala Likert de 7 puntos, que varía entre 1 «completamente en desacuerdo» y 7 «completamente de acuerdo». Los resultados expresan la posición de los psicoterapeutas en 5 dimensiones que componen el EPT: instruccional, expresiva, involucración, atencional y operativa. Estas dimensiones, respectivamente, presentan en la versión original unos índices de fiabilidad alfa de Cronbach de .69, $.75, .75, .80$ y .76 .

\section{Procedimiento}

El reclutamiento de los participantes se llevó a cabo por muestreo incidental no probabilístico, entre el $28 \mathrm{de}$ noviembre y el 31 de diciembre de 2018, a través de una batería de cuestionarios que se administraron on-line mediante la plataforma SurveyMonkey. Se contactaron por e-mail un total de 1283 psicólogos clínicos (incluidos residentes y especialistas) mediante los canales de comunicación internos de la Asociación Nacional de Psicólogos Clínicos y Residentes (ANPIR), la Sociedad de Psicología Clínica Asturiana (SOPCA) y la Asociación Galega de Psicólogos Internos Residentes (AGAPIR). Asumiendo un 5\% de error, para obtener un tamaño muestral representativo de la población objeto de estudio era preciso obtener una $n \geq 297$. Se completó un consentimiento informado, se garantizó la confidencialidad de los participantes, el uso de sus respuestas exclusivamente para fines de investigación y no recibieron compensación alguna por su participación.

El presente estudio forma parte del proyecto Personal therapist style \& psychotherapists' attitudes and use of research outcomes in their clinical practice [Estilo personal del terapeuta y actitudes de los psicoterapeutas y uso de los resultados de la investigación en su práctica clínica]. Se inscribe en el marco general de la Investigación Orientada por la Práctica (Castonguay y Muran, 2015), un movimiento internacional que persigue fomentar y vertebrar redes de colaboración entre investigadores y clínicos para el desarrollo de programas científicos. Se trata de un proyecto independiente y no financiado, pero cuenta con el apoyo institucional de ANPIR (España) y la Fundación Aiglé (Argentina).

\section{Análisis estadísticos}

La literatura muestra que cuando el EPT-C ha sido sometido a estudio emergen un total de cinco modelos o 
soluciones factoriales (Casari, Morán y Isón, 2017). En este trabajo se han analizado los cinco modelos existentes hasta encontrar la solución con mejores indicadores de ajuste y fiabilidad. Para ello, se ha procedido mediante la técnica del Análisis Factorial Confirmatorio (AFC) que se fundamenta en la Teoría Clásica de los Tests (DeVellis, 2006). Los modelos del AFC se calcularon mediante el método de máxima verosimilitud aplicando correcciones robustas a través del programa EQS v. 6.1. No existen datos perdidos porque el uso de una plataforma online de recolección de datos nos permitió obligar a completar cada respuesta para poder avanzar hasta finalizar el cuestionario.

El primer modelo puesto a prueba fue la solución original de 36 ítems y 5 factores encontrada en terapeutas argentinos (Fernández-Álvarez et al., 2003). El segundo modelo fue la versión abreviada del primer modelo que mantiene los 5 factores con 21 ítems también en una muestra argentina (Castañeiras et al., 2008). El tercer modelo fue la propuesta de Quiñones, Melipillán y Ramírez (2010) que conserva los 36 ítems del original, pero organizados en torno a 4 factores, tras realizar un análisis factorial exploratorio en una muestra chilena. El cuarto modelo se analizó por coherencia con la literatura sobre el constructo (Casari et al., 2017), pero como señalan los propios autores no es estrictamente un modelo diferente de la versión original. Consiste en unos resultados similares a los encontrados en la muestra chilena, donde las funciones atencional y operativa aparecen subsumidas en un mismo factor. Esta comunalidad ya se encontró en la primera validación, pero ambos factores se mantuvieron separados por criterios teóricos (Fernández-Álvarez et al., 2003; 2005). Finalmente, el quinto modelo analizado fue la versión portuguesa (Moura, Corbella y Mena, 2011) que confirma el modelo de Castañeiras et al. (2008) con la única diferencia de mantener 20 ítems tras eliminar el ítem 33 («Procuro dirigir mi atención a la totalidad de lo que pasa en las sesiones») que no mostró una carga factorial adecuada.

El ajuste de los distintos modelos se evaluó utilizando la prueba de chi-cuadrado, el índice de ajuste comparativo (CFI), el índice de bondad del ajuste (GFI) y el error cuadrático medio de aproximación (RMSEA). El criterio de buen ajuste empleado consistió en establecer como puntuaciones de corte valores iguales o superiores a .90 para CFI y GFI y valores inferiores a .08 para RMSEA (Marsh, Hau y Wen, 2004). Por último, mediante el programa SPSS v. 22, se analizó la fiabilidad de los factores que componen el EPT-C calculando sus índices de consistencia interna (alfa de Cronbach) y se estudiaron las correlaciones entre los factores.

\section{Resultados}

Los resultados del AFC muestran que los modelos 2 (Castañeiras et al., 2008) y 5 (Moura et al., 2011) presentaron los mejores índices de ajuste. Como se puede ver en la Tabla 2, ambos modelos obtuvieron un CFI de .90 , valores en GFI cercanos a .90 , una reducción estadísticamente significativa del valor chi cuadrado respecto a los otros modelos y un valor RMSEA inferior a .06 (Hu y Bentler, 1998).

Tabla 2. Índices de ajuste de los 5 modelos contrastados del EPT-C y de los 2 modelos reestimados en este estudio

\begin{tabular}{lcccccc}
\hline & $\chi^{2}$ & gl & CFI & GFI & RMSEA & $90 \%$ IC \\
\hline Modelo 1 & 4393.87 & 630 & .83 & .65 & .057 & $(.052, .061)$ \\
Modelo 2 & 1650.54 & 210 & .90 & .86 & .048 & $(.039, .056)$ \\
Modelo 3 & 4393.87 & 630 & .80 & .66 & .060 & $(.056, .064)$ \\
Modelo 4 & 4393.87 & 630 & .79 & .65 & .061 & $(.057, .065)$ \\
Modelo 5 & 1537.24 & 190 & .90 & .87 & .048 & $(.039, .057)$ \\
Modelo 6 & $\mathbf{1 5 9 6 . 3 3}$ & $\mathbf{1 9 0}$ & $\mathbf{. 9 1}$ & $\mathbf{. 8 7}$ & $\mathbf{. 0 4 7}$ & $\mathbf{( . 0 3 8 , . 0 5 6 )}$ \\
Modelo 7 & 1493.43 & 171 & .91 & .88 & .047 & $(.038, .057)$ \\
\hline
\end{tabular}

Notas: $\chi^{2}=$ prueba Chi-cuadrado; gl = grados de libertad; CFI = índice de ajuste comparativo; GFI = índice de bondad del ajuste; RMSEA = error cuadrático medio de aproximación; IC = intervalo de confianza del estadístico RMSEA.

Sin embargo, tras analizar las cargas factoriales de los ítems, se encontró que el ítem 28 («Si algo me irrita durante una sesión, puedo expresarlo») no presenta una carga factorial adecuada (.26) en los modelos 2 y 5 . En consecuencia, se reestimaron ambos modelos excluyendo el ítem 28 de los análisis. Como resultado aparecen en la Tabla 2 los modelos 6 y 7 que se corresponden respectivamente con la reestimación de los modelos 2 y 5 . Ambos modelos presentaron, a su vez, unos índices de ajuste aceptables. Por otro lado, cuando se analizaron los modelos 2 y 6 se observó una carga factorial adecuada para el ítem 33 (Tabla 3), a diferencia de lo que se halló en el estudio de Moura et al. (2011).

En la tabla 3 se presentan las cargas factoriales y los estadísticos descriptivos que constituyen el modelo 6, compuesto por 20 ítems tras suprimir el ítem 28 y mantener el 33. Todos los ítems presentan una carga factorial adecuada con valores que oscilan entre .38 y .71 . En apoyo de esta solución cabe destacar que el ítem 28 ya presentó un peso factorial inadecuado (.27) en el trabajo de Moura et al. (2011), pero los autores lo mantuvieron por motivos que no se justifican en el trabajo de referencia. En cualquier caso, dicho dato parece apoyar que aquí se opte por el modelo reestimado número 6 , cuya 
Tabla 3. Cargas factoriales y estadísticos descriptivos de los 20 ítems del EPT-C

\begin{tabular}{rcrccc}
\hline Ítem & Carga M6 & Media & DT & Asimetría & Curtosis \\
\hline 1 & .44 & 5.28 & 1.38 & -1.02 & .30 \\
3 & .68 & 2.84 & 1.41 & .70 & -.47 \\
6 & .38 & 4.23 & 1.28 & -.24 & -1.08 \\
9 & .39 & 3.58 & 1.60 & .05 & -.55 \\
10 & .39 & 3.41 & 1.52 & .17 & -.91 \\
11 & .48 & 5.91 & 0.99 & -1.19 & 2.44 \\
13 & .71 & 3.88 & 1.70 & .23 & -1.28 \\
15 & .67 & 4.33 & 1.37 & -.35 & -.37 \\
18 & .53 & 5.19 & 1.34 & -.99 & .61 \\
21 & .45 & 3.82 & 1.44 & .45 & -.80 \\
22 & .63 & 3.20 & 1.44 & .47 & -.90 \\
23 & .60 & 4.74 & 1.50 & -.66 & -.47 \\
25 & .55 & 2.40 & 1.45 & 1.33 & 1.26 \\
27 & .63 & 2.86 & 1.55 & .95 & .13 \\
29 & .42 & 4.04 & 1.52 & -.30 & -1.05 \\
31 & .71 & 2.51 & 1.43 & 1.03 & .48 \\
33 & .41 & 5.48 & 1.17 & -1.14 & 1.11 \\
34 & .69 & 4.33 & 1.44 & -.52 & -.70 \\
35 & .56 & 3.22 & 1.27 & .41 & -.93 \\
36 & .44 & 4.02 & 1.52 & -.16 & -.87 \\
\hline
\end{tabular}

Notas. M6 = Modelo 6; DT = Desviación típica.

solución factorial se dibuja en la Figura 1. Dicha Figura presenta un modelo compuesto por 20 ítems que se organizan en torno a la estructura clásica pentafactorial y muestra las cargas factoriales de los ítems y las inter-correlaciones entre factores.

Respecto a la fiabilidad de los diferentes factores que componen el EPT-C se encontraron unos valores alfa de Cronbach de .54 para las dimensiones instruccional y expresiva, de .72 para involucración y operativa, y de .47 para el factor atencional. El análisis ítem por ítem reveló que de suprimirse el ítem 33, el valor alfa de Cronbach del factor atencional descendía a .41. Lo cual redunda en optar por el modelo 6 para la muestra del presente estudio. Dichos valores son coherentes con los encontrados en otros trabajos sobre el EPT-C (Casari et al., 2017; 2018; Moura et al., 2011).

Por último, en la Tabla 4 aparecen las correlaciones entre los distintos factores que componen el EPT-C. Se encontraron correlaciones estadísticamente significativas: de signo positivo entre las dimensiones atencional y operativa, así como entre involucración y expresiva; y de signo negativo para las relaciones entre las dimensiones

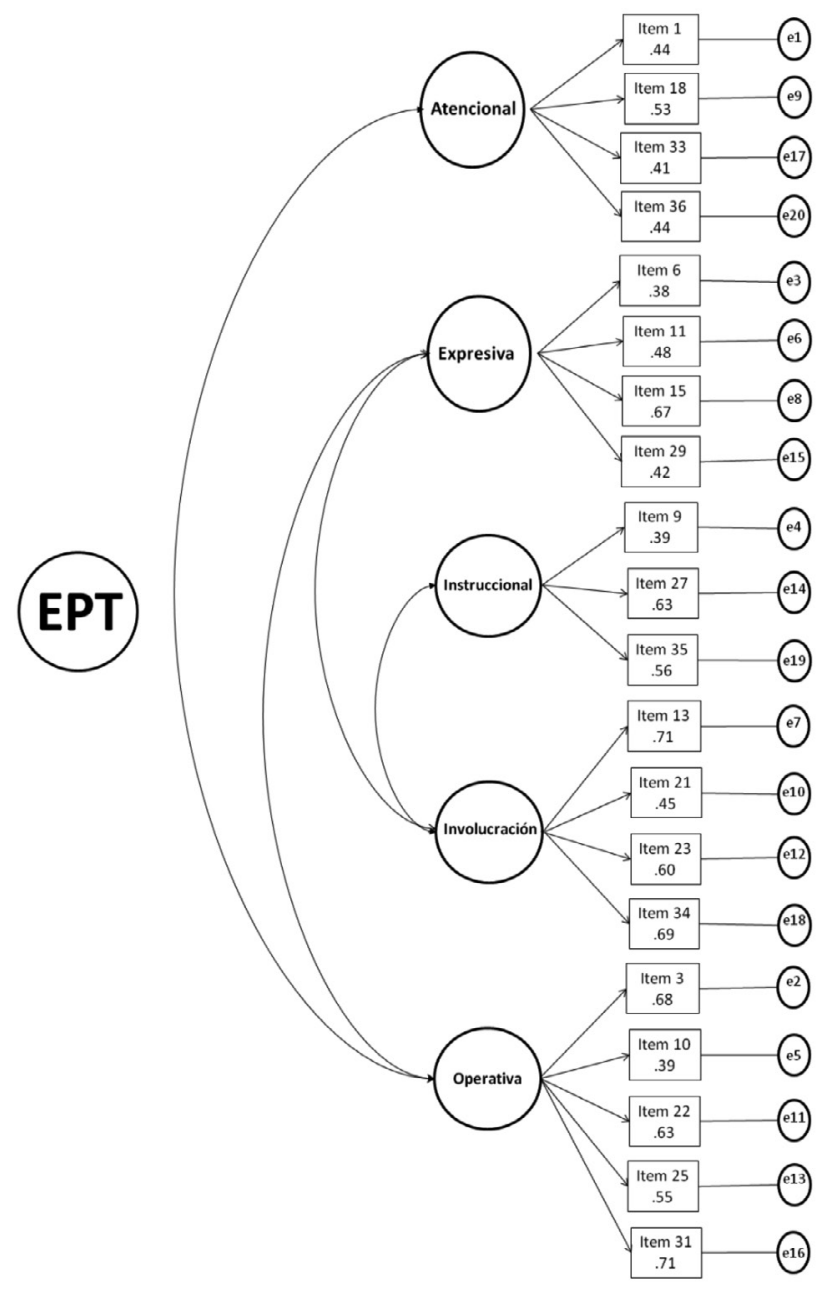

Figura 1. Estructura factorial del modelo reestimado de 20 ítems y 5 factores

operativa y atencional con las dimensiones expresiva e involucración y entre los factores involucración e instruccional.

Tabla 4. Matriz de correlaciones entre los diferentes factores del EPT-C

\begin{tabular}{|c|c|c|c|c|}
\hline & Expresiva & Instruccional & Involucración & Operativa \\
\hline Atencional & $-.19^{* *}$ & .01 & $-.12^{*}$ & $.40^{* *}$ \\
\hline Expresiva & & -.03 & $.17^{* *}$ & $-.17^{* *}$ \\
\hline Instruccional & & & $-.14^{* *}$ & .09 \\
\hline Involucración & & & & $-.12^{*}$ \\
\hline
\end{tabular}

Notas: ${ }^{*} p<.05 ;{ }^{* *} p<.001$

\section{Discusión}

El objetivo principal del presente estudio consistió en evaluar las propiedades psicométricas y la estructura factorial del EPT-C en una muestra de psicólogos clíni- 
cos españoles. El EPT-C es un auto-informe que cuenta con más de 20 años de tradición investigadora y que todavía no había sido estudiado psicométricamente en nuestro contexto profesional y socio-cultural. Como objetivos más específicos deseábamos: (1) examinar las propuestas originales en una muestra representativa de nuestra práctica clínica y (2) dirimir qué versión de las disponibles era la más recomendable para usos formativos y de investigación en nuestro entorno inmediato.

En relación al primer objetivo, mediante la técnica del AFC se estudiaron las cinco soluciones factoriales encontradas en la literatura empírica (Casari et al., 2017). Los resultados mostraron que los dos modelos abreviados de la versión original (Castañeiras et al., 2008; Moura et al., 2011) presentaron los mejores índices de ajuste, de consistencia interna y conservaron la estructura pentafactorial clásica. No obstante, a diferencia de dichos trabajos, en nuestro estudio los ítems 28 y 33 presentaron cargas factoriales que obligaban a hacer una reestimación de los citados modelos. A diferencia del estudio de Moura et al. (2011) se propuso mantener el ítem 33 por presentar una carga factorial adecuada y, a diferencia de Castañeiras et al. (2008), se propuso eliminar el ítem 28 por presentar un peso factorial inferior a .30. La reestimación de ambos modelos, excluyendo el ítem 28, mejoró los índices de ajuste de ambos modelos (Tabla 2). Los resultados de este estudio también se apoyaron en el análisis de la consistencia interna de los 5 factores que componen el EPT-C y en las correlaciones de cada ítem con su respectivo factor de referencia. De este modo, este trabajo es coherente con la investigación previa que orienta en un mejor ajuste de los modelos abreviados respecto a los de 36 ítems (Casari et al., 2017; Moura et al., 2011) y apoya parcialmente la solución de 21 ítems encontrada por Castañeiras et al. (2008). Sin embargo, como ya sucedió en la validación portuguesa con el ítem 33, en este caso parece razonable optar por una solución de 20 reactivos tras eliminar el ítem 28. El modelo propuesto presenta unas propiedades psicométricas adecuadas, es coherente con la estructura pentafactorial planteada por los autores originales y conserva el mayor número de ítems e información posibles sobre variables estilísticas del terapeuta. En este sentido, respecto al segundo objetivo, en el Anexo I se adjunta la versión adaptada que ha resultado de este proceso de validación del EPT-C.

Respecto a las correlaciones entre factores, éstas fueron pequeñas salvo para la relación entre las dimensiones atencional y operativa que fue moderada (Tabla 4). En cualquier caso, con pequeñas variaciones, los datos se comportaron en la dirección esperada y fueron coherentes con la literatura empírica sobre el constructo (p.ej., Casari et al., 2017; 2018). Sin embargo, es oportuno realzar que el hecho de que existan relaciones estadísticamente significativas entre los diversos factores que componen el EPT-C ejemplifica la dificultad que entraña separar en partes estrictas variables estilísticas que, ciertamente, operan como un todo armónico y entrelazado (Prado-Abril et al., 2017). Asimismo, la mayor correlación encontrada entre las funciones atencional y operativa (.40) aviva nuevamente el debate en torno a si ambos factores son aspectos diferenciados o facetas de un mismo factor de orden superior (Casari et al., 2017; Fernández-Álvarez et al., 2003; 2005; Quiñones et al., 2010).

En suma, este estudio aporta evidencia preliminar en nuestro entorno de un instrumento que permite medir una de las variables clave del proceso psicoterapéutico: la persona del terapeuta. En el contexto contemporáneo de la práctica basada en la evidencia (Prado-Abril et al., 2017; 2019), evaluar al clínico es tan necesario como evaluar las características de los pacientes (p.ej., Gimeno-Peón et al., 2018; Pineda, Valiente, Chorot, Piqueras y Sandín, 2018; Sandín, Valiente, Pineda, García-Escalera, y Chorot, 2018) y la relación que se establece entre ambos (p.ej., Andrade-González y Fernández-Liria, 2015). Por otro lado, hasta donde tenemos constancia, se trata del primer estudio que analiza en nuestro país una muestra amplia de psicólogos clínicos debidamente acreditados. Por último, se normaliza en nuestro contexto profesional y socio-cultural un cuestionario de prestigio internacional que surge de una filosofía investigadora y asistencial que persigue cubrir la brecha entre investigación y asistencia, entre científicos y clínicos, promoviendo el modelo del clínico como científico-practicante bajo el marco de la investigación orientada por la práctica (Castonguay y Muran, 2015; Fernández-Álvarez, Gómez y García, 2015b).

Antes de concluir deben ponerse de relieve las diversas limitaciones que posee el estudio. En primer lugar, el uso de una técnica de muestreo no probabilístico afecta a las posibilidades de generalización de los resultados. A este respecto, es pertinente subrayar que no existen datos ni fuentes fiables que orienten en el tamaño del universo de psicólogos clínicos que existen en la actualidad en nuestro país. En el futuro, estos defectos se podrían mitigar si finalmente el Ministerio de Sanidad es capaz de avanzar en la creación del Registro Estatal de Profesionales Sanitarios (Real Decreto 640/2014; Orden SSI/890/2017). Una propuesta legislativa que cuenta con más de 15 años sin regulación efectiva y que, de materializarse, permitiría garantizar la transparencia sobre la cualificación de los profesionales para conocimiento de los ciudadanos, al tiempo que supondría disponer de un 
censo que facilitaría el estudio fiable del universo profesional. Una segunda limitación de este estudio, a pesar de que se procedió mediante canales de distribución cerrados que garantizan las características de la muestra, reside en el uso de una metodología de evaluación on-line que posee inconvenientes respecto a los procedimientos tradicionales (Wright, 2006). Finalmente, el uso de una medida de auto-informe obliga a recalcar que lo evaluado en este trabajo puede versar más sobre la representación mental que los clínicos tienen de su estilo personal que con lo que efectivamente actúan a nivel conductual. En este sentido, en futuras investigaciones será imprescindible combinar los datos auto-informados con la metodología observacional y el criterio de jueces externos para incrementar la validez de constructo del EPT-C (Fernández-Álvarez et al., 2017). Como corolario de este trabajo, cabe enfatizar que serán futuras investigaciones las que determinarán si este proyecto servirá para estimular a otros científicos-practicantes de nuestro entorno a virar el foco del énfasis actual en los factores técnicos para investigar también, con seriedad y rigor, otras variables humanas que gozan de apoyo empírico como los efectos del terapeuta (Barkham et al., 2017). No en vano, la psicoterapia sigue siendo un particular encuentro, en un contexto dado, de carácter invariablemente interpersonal.

\section{Conflictos de intereses}

Los autores declaran que no existen conflictos de intereses.

\section{Referencias}

American Psychological Association [APA] (2013). Recognition of psychotherapy effectiveness. Psychotherapy, 50, 102-109. doi:10.1037/a0030276

Andrade-González, N., y Fernández-Liria, A. (2015). Spanish adaptation of the Working Alliance Inventory (WAI). Psychometric properties of the patient and therapist forms (WAI-P and WAI-T). Anales de Psicologia, 31, 524-533. doi:10.6018/analesps.31.2.177961

Barkham, M., Lutz, W., Lambert, M. J., y Saxon, D. (2017). Therapist effects, effective therapists, and the law of variability. In L. G. Castonguay, y C. E. Hill (Eds.), How and why are some therapists better than others? (pp. 13-36). Washington, DC: American Psychological Association.

Beutler, L. E., Malik, M., Alimohamed, S., Harwood, T. M., Talebi, H., Noble, S., y Wong, E. (2004). Therapist variables. In M. J. Lambert (Ed.), Bergin and Garfield's handbook of psychotherapy and behavior change ( $5^{\text {th }}$ Ed., pp. 227-306). Hoboken, NJ: Wiley.

Bohart, A. C., y Wade, A. G. (2013). The client in psychotherapy. In M. J. Lambert (Ed.), Bergin and Garfield's handbook of psychotherapy and behavior change (6 $6^{\text {th }}$ Ed., pp. 219-257). Hoboken, NJ: Wiley.

Casari, L. M., Albanesi, S. y Maristany, M (2013). Influencia del enfoque teórico en el estilo personal del terapeuta. Psicogente, 16, 132-142.

Casari, L. M., Ison, M. y Gómez, B. (2018). Estilo Personal del Terapeuta: Estado actual (1998-2017). Revista Argentina de Clínica Psicológica, 27, 466-477. doi: 10.24205/03276716. 2018.1082

Casari, L. M., Morán, V. y Ison, M. (2017). Cuestionario de estilo personal del terapeuta: Análisis factorial confirmatorio de modelos rivales con psicoterapeutas argentinos. Psychologia, 11, 69-84. doi: 10.21500/19002386.2725

Castañeiras, C., García, F., Lo Bianco, J., y Fernández-Álvarez, H. (2006). Modulating effect of experience and theoreticaltechnical orientation on the personal style of the therapist. Psychotherapy Research, 16, 587-593. doi:10.1080/ 10503300600802867

Castañeiras, C., Ledesma, R., García, F. y Fernández-Álvarez, H. (2008). Evaluación del Estilo Personal del Terapeuta: Presentación de una versión abreviada del Cuestionario EPT-C. Terapia Psicológica, 26, 5-13. doi:10.4067/S071848082008000100001

Castonguay, L. G., y Muran, J. C. (2015). Fostering collaboration between researchers and clinicians through building practiceoriented research: An introduction. Psychotherapy Research, 25, 1-5. doi:10.1080/10503307.2014.966348

DeVellis, R. F. (2006). Classical test theory. Medical Care, 44, S50-S59. doi: 10.1097/01.mlr.0000245426.10853.30

Fernández-Álvarez, H., Castañeiras, C., Curtarelli, A., García, F., Gómez, B., Lichtenberger, A. y Corbella, S. (2015a). Presentación de una Guía para la Observación y Clasificación de la Conducta Verbal de los Terapeutas. Terapia Psicológica, 33, 23-34. doi:10.4067/S0718-48082015000100003

Fernández-Álvarez, H., García, F., Castañeiras, C. y Rial, V. (2005). Normalización del Cuestionario de Evaluación sobre El estilo Personal del Terapeuta (EPT-C) en una población de psicoterapeutas de Argentina. Presentación realizada en las XII Jornadas de Investigación de la Facultad de Psicología de la Universidad de Buenos Aires. Buenos Aires, Argentina.

Fernández-Álvarez, H., García, F., Lo Bianco, J., y Corbella, S. (2003). Assessment Questionnaire on the Personal Style of the Therapist PST-Q. Clinical Psychology and Psychotherapy, 10, 116-125. doi:10.1002/cpp.358

Fernández-Álvarez, H., García, F., y Scherb, E. (1998). The research program at AIGLE. Journal of Clinical Psychology, 54, 343-359. doi:10.1002/(SICI)1097-4679(199804)54:3 $<343::$ AID-JCLP5>3.0.CO;2-Q

Fernández-Alvarez, H., Gómez, B., y García, F. (2015b). Bridging the gap between research and practice in a clinical and training network: Aigle's Program. Psychotherapy Research, 25, 8494. doi: 10.1080/10503307.2013.856047

Fernández-Álvarez, J., Castañeiras, C., García, F., Gómez, B. y Fernández-Álvarez, H. (2017). Investigando el estilo personal del terapeuta: Correspondencia entre las autodescripciones y la observación de jueces externos. Revista de Psicopatología y Psicología Clínica, 22, 207-217. doi:10.5944/rppc.vol.22. num.3.2017.18238 
Gimeno-Peón, A., Barrio-Nespereira, A. y Prado-Abril, J. (2018). Monitorización sistemática y feedback en psicoterapia. Papeles del Psicólogo, 39, 174-182. doi:10.23923/pap.psicol 2018.2872

Gómez, B., Castañeiras, C., Curtarelli, A., Fraga Míguez, M., García, F., Maristany, M., y Fernández-Álvarez, H. (2011). Personal Style of the Therapist (PST). Intra and inter therapist verbal behavior analysis. How do therapists ask questions? Póster presentado en el $42^{\text {nd }}$ Annual Meeting of the Society for Psychotherapy Research. Berna, Suiza.

Hofmann, S. G., y Barlow, D. H. (2014). Evidence-based psychological interventions and the common factors approach: The beginnings of a rapprochement? Psychotherapy, 51, 510513. doi: $10.1037 / \mathrm{a} 0037045$

Hu, L.-t., y Bentler, P. M. (1998). Fit indices in covariance structure modeling: Sensitivity to underparameterized model misspecification. Psychological Methods, 3, 424-453. doi:10.1037/1082-989X.3.4.424

Lambert, M. J. (2013). The efficacy and effectiveness of psychotherapy. In M. J. Lambert (Ed.), Bergin and Garfield's handbook of psychotherapy and behavior change ( $6^{\text {th }} \mathrm{Ed}$., pp. 169-218). Hoboken, NJ: Wiley.

Laska, K. M., Gurman, A. S., y Wampold, B. E. (2014). Expanding the lens of Evidence-based practice in psychotherapy: A common factors perspective. Psychotherapy, 51, 467-481. doi: $10.1037 / \mathrm{a} 0034332$

Lee, J. A., Neimeyer, G. J., y Rice, K. G. (2013). The relationship between therapist epistemology, therapy style, working alliance, and interventions use. American Journal of Psychotherapy, 67, 323-345.

Marsh, H. W., Hau, K.-T., y Wen, Z. (2004). In search of golden rule: Comment on hypothesis testing approaches to setting cutoff values for fit indexes and danger in overgeneralizing $\mathrm{Hu}$ and Bentler's (1999) Findings. Structural Equation Modeling: A Multidisciplinary Journal, 11, 320-341. doi: 10.1207/ s15328007sem1103_2

Moura, H., Corbella, S. y Mena, P. (2011). Análisis factorial confirmatorio de la Versión Corta Portuguesa del Cuestionario del Estilo Personal del Terapeuta (EPT-C). Revista Argentina de Clínica Psicológica, 20, 91-98.

Norcross, J. C., y Goldfried, M. R. (Eds.) (2005). Handbook of Psychotherapy Integration (2 $2^{\text {nd }}$ Ed.). New York: Oxford University Press.

Orlinsky, D. E., Grawe, K., y Parks, B. K. (1994). Process and outcome in psychotherapy. Noch einmal. In A. E. Bergin, y S.
L. Garfield (Eds.), Handbook of psychotherapy and behavior change (4 ${ }^{\text {th }}$ Ed., pp. 270-376). New York: Wiley.

Pineda, D., Valiente, R. M., Chorot, P., Piqueras, J. A. y Sandín, B. (2018). Invarianza factorial y temporal del Cuestionario de Regulación Emocional (ERQ). Revista de Psicopatología y Psicología Clínica, 23, 109-120. doi: 10.5944/rppc.vol.23. num.2.2018.21823

Prado-Abril, J., Gimeno-Peón, A., Inchausti, F. y Sánchez-Reales, S. (2019). Pericia, efectos del terapeuta y práctica deliberada: El ciclo de la excelencia. Papeles del Psicólogo, 40, 89-100. doi:10.23923/pap.psicol2019.2888

Prado-Abril, J., Sánchez-Reales, S. y García-Campayo, J. (2016). Dificultades en el manejo cognitivo-interpersonal del trastorno narcisista de la personalidad: Estudio de caso. Revista Argentina de Clínica Psicológica, 25, 317-325.

Prado-Abril, J., Sánchez-Reales, S. y Inchausti, F. (2017). En busca de nuestra mejor versión: Pericia y excelencia en Psicología Clínica. Ansiedad y Estrés, 23, 110-117. doi:10.1016/j.anyes.2017.06.001

Quiñones, A., Melipillán, R. y Ramírez, P. (2010). Estudio psicométrico del Cuestionario de Evaluación del Estilo Personal del Terapeuta (EPT-C) en psicoterapeutas acreditados en Chile. Revista Argentina de Clínica Psicológica, 19, 273-281.

Quiñones, A., Ugarte, C., Ceric, F., García, F. y Santibañez, P. (2019). Estilo Personal del Terapeuta: Comparación ente terapeutas cognitivos post-racionalistas y sistémicos. Revista Argentina de Clínica Psicológica, 28, 48-55. doi: 10.24205/03276716.2018.1054

Sandín, B., Valiente, R. M., Pineda, D., García-Escalera, J. y Chorot, P. (2018). Escala de síntomas de los Trastornos de Ansiedad y Depresión (ESTAD): Datos preliminares sobre su estructura factorial y sus propiedades psicométricas. Revista de Psicopatología y Psicología Clínica, 23, 163-177. doi: 10.5944/rppc.vol.23.num.3.2018.22976

Vides-Porras, A., Grazioso, M. y García de la Cadena, C. (2012). El estilo personal del terapeuta guatemalteco en el interior del país. Revista Argentina de Clínica Psicológica, 21, 33-43.

Wampold, B. E., y Imel, Z. E. (2015). The great psychotherapy debate: The research evidence for what works in psychotherapy $\left(2^{\text {nd }}\right.$ Ed.). New York, NY: Routledge.

Wright, K. B. (2006). Researching Internet-based populations: Advantages and disadvantages of online survey research, online questionnaire authoring software packages, and web survey services. Journal of Computer-Mediated Communication, 10, 00-00. doi: 10.1111/j.1083-6101.2005.tb00259.x 


\section{$\Delta \underline{A G L E E}$}

\section{Cuestionario de Evaluación del Estilo Personal del Terapeuta (EPT-C)}

El presente inventario está destinado a conocer su estilo personal como terapeuta. No hay respuestas buenas o malas ya que diferentes estilos pueden ser igualmente beneficiosos. Más allá de que su labor varía con los diferentes pacientes, queremos que responda según sea su modo más general y frecuente de llevar adelante su trabajo. No piense demasiado en el significado de cada afirmación. La respuesta más espontánea es la más valiosa.

Completamente en desacuerdo // $1-2-3-4-5-6-7 \$ Completamente de acuerdo

\begin{tabular}{|c|c|}
\hline 1 & Suelo mantener mi escucha más bien abierta y receptiva más que concentrada y focalizada \\
\hline 2 & Como terapeuta prefiero indicar a los pacientes qué debe hacerse en cada sesión \\
\hline 3 & Las emociones que me despierta el paciente son decisivas para el curso del tratamiento \\
\hline 4 & Tiendo a exigir cumplimiento estricto con los honorarios \\
\hline 5 & Los tratamientos pautados me merecen escasa valoración \\
\hline 6 & La expresión de las emociones es un poderoso instrumento de cambio \\
\hline 7 & Fuera de las horas de trabajo dejo de pensar en los pacientes \\
\hline 8 & Los verdaderos cambios se producen en el curso de sesiones con un clima emocional intenso \\
\hline 9 & Me gusta sentirme sorprendido por el material del paciente sin tener ideas previas \\
\hline 10 & Lo que les pasa a mis pacientes tiene poca influencia en mi vida personal \\
\hline 11 & En mis intervenciones soy predominantemente directivo \\
\hline 12 & Pienso bastante en mi trabajo, aún en mis horas libres \\
\hline 13 & Puedo planear un tratamiento en su totalidad desde que se inicia \\
\hline 14 & Nunca modifico la duración de las sesiones, a menos que sea imprescindible \\
\hline 15 & Una alta proximidad emocional es imprescindible para promover cambios terapéuticos \\
\hline 16 & Prefiero los tratamientos donde están programados todos los pasos a seguir \\
\hline 17 & Procuro dirigir mi atención a la totalidad de lo que pasa en las sesiones \\
\hline 18 & Tengo en mente los problemas de los pacientes más allá de las sesiones \\
\hline 19 & Soy bastante laxo con los horarios \\
\hline 20 & Desde el comienzo de las sesiones me dispongo a dejar flotar mi atención \\
\hline
\end{tabular}

Notas: Reproducido con permiso. Los valores de los ítems señalados con * se puntúan de forma inversa. Clave de corrección: Instruccional $=\Sigma 4,14,19^{*} ;$ Expresiva $=\Sigma 3,6,8,15 ;$ Involucración $=\Sigma 7^{*}, 10^{*}, 12,18 ;$ Atencional $=\Sigma 1^{*}, 9^{*}, 17^{*}, 20^{*} ;$ Operativa $=\Sigma 2,5^{*}, 11,13$, 16. 